\title{
COMPLEX HADAMARD MATRICES ATTACHED TO A 3-CLASS NONSYMMETRIC ASSOCIATION SCHEME
}

\author{
TAKUYA IKUTA AND AKIHIRO MUNEMASA
}

\begin{abstract}
In this paper we classify complex Hadamard matrices contained in the Bose-Mesner algebra of nonsymmetric 3-class association schemes. As a consequence of our classification, we have two infinite families and some small examples of complex Hadamard matrices contained in the Bose-Mesner algebra of a self-dual fission of a complete multipartite graph.
\end{abstract}

\section{INTRODUCTION}

A complex Hadamard matrix is a square matrix $W$ of order $n$ which satisfies $W \bar{W}^{\top}=n I$ and all of whose entries are complex numbers of absolute value 1. A complex Hadamard matrix is said to be Butson-type, if all of its entries are roots of unity. Complex Hadamard matrices which are not of Butson-type but are constructed combinatorially, found applications outside combinatorics [15]. In particular, a family of complex Hadamard matrices has been found in the BoseMesner algebras of nonsymmetric 2-class association schemes [13. In our earlier work [8], we considered nonsymmetric hermitian complex Hadamard matrices belonging to the Bose-Mesner algebra of a nonsymmetric 3-class association scheme $\mathfrak{X}=\left(X,\left\{R_{i}\right\}_{i=0}^{3}\right)$, where $R_{1}^{\top}=R_{2}, R_{3}$ symmetric. Its first eigenmatrix is given by

$$
P=\left(\begin{array}{cccc}
1 & \frac{k_{1}}{2} & \frac{k_{1}}{2} & k_{2} \\
1 & \frac{1}{2}(r+b i) & \frac{1}{2}(r-b i) & -(r+1) \\
1 & \frac{1}{2}(r-b i) & \frac{1}{2}(r+b i) & -(r+1) \\
1 & \frac{s}{2} & \frac{s}{2} & -(s+1)
\end{array}\right),
$$

where $k_{1}$ is an even positive integer, $r, s$ are integers, $b$ is a positive real number, and $i^{2}=-1$ (see Lemma 4 for more precise information.). Let $\mathfrak{A}$ be the Bose-Mesner algebra of $\mathfrak{X}$ which is the linear span of the adjacency matrices $A_{0}, A_{1}, A_{2}, A_{3}$ of $\mathfrak{X}$, where $A_{1}^{\top}=A_{2}, A_{3}$ symmetric. Let $w_{1}, w_{2}, w_{3}$ be complex numbers of absolute value 1 . We assume that $w_{1} \neq w_{2}$, and set

$$
W=A_{0}+w_{1} A_{1}+w_{2} A_{2}+w_{3} A_{3} \in \mathfrak{A} .
$$

In [8, Theorem 1], we have shown the following: We assume that the matrix (2) is a hermitian complex Hadamard matrix and not a real Hadamard matrix. Then $\mathfrak{X}$ is a nonsymmetric association scheme whose unique nontrivial symmetric relation

Date: April 19, 2019.

2010 Mathematics Subject Classification. 05E30,05B30.

Key words and phrases. association scheme, complex Hadamard matrix.

This work was supported by JSPS KAKENHI grant number 17K05155. 
consists of $2 \alpha$ cliques of size $2 \alpha$, and the first eigenmatrix of $\mathfrak{X}$ is given by

$$
\left(\begin{array}{cccc}
1 & \alpha(2 \alpha-1) & \alpha(2 \alpha-1) & 2 \alpha-1 \\
1 & \alpha i & -\alpha i & -1 \\
1 & -\alpha i & \alpha i & -1 \\
1 & -\alpha & -\alpha & 2 \alpha-1
\end{array}\right),
$$

where $\alpha$ is a positive integer. Moreover, $w_{1}= \pm i$ and $w_{3}=1$. Note that we have assumed $w_{1} \neq w_{2}$ since, if $w_{1}=w_{2}$, then $W$ belongs to the Bose-Mesner algebra of a strongly regular graph. Such complex Hadamard matrices, or more generally, type-II matrices, have been classified by Chan and Godsil [4]. Therefore, we will assume $w_{1} \neq w_{2}$ throughout this paper.

As a natural problem, omitting the hermitian condition, we are interested in whether other complex Hadamard matrices arise or not. In this paper, we show that such complex Hadamard matrices are contained in the Bose-Mesner algebra of a self-dual fission of a complete multipartite graph. More precisely, we have two infinite families and some small examples, as given in the following main theorem.

Theorem 1. With the above assumptions, the matrix (2) is a complex Hadamard matrix if and only if $\left(k_{1}, k_{2}, r, s, b\right)=(2 a(2 a-1) c, 2 a-1,0,-2 a, 2 a \sqrt{c})$ for some positive integers $a, c$, and one of the following holds.

(i) $c=1$, and

(a) $\left(w_{1}, w_{2}, w_{3}\right)=(w,-w, 1)$ with $|w|=1$,

(b) $\left(w_{1}, w_{2}, w_{3}\right)=\left(w_{ \pm}, w_{\mp}, w_{ \pm} w_{\mp}\right)$, where

$$
w_{ \pm}=\frac{-(a-1) \mp a \sqrt{2 a(a-1)}+(-a \pm(a-1) \sqrt{2 a(a-1)}) i}{2 a^{2}-2 a+1},
$$

(c) $a=2,\left(w_{1}, w_{2}, w_{3}\right)=\left(\frac{3 \pm 4 i}{5},-1, \frac{-3 \mp 4 i}{5}\right),\left(-1, \frac{3 \pm 4 i}{5}, \frac{-3 \mp 4 i}{5}\right)$,

(ii) $a=1, c=3$, and

(d) $\left(w_{1}, w_{2}, w_{3}\right)=\left(\frac{1 \pm 2 \sqrt{2} i}{3},-1,1\right),\left(-1, \frac{1 \pm 2 \sqrt{2} i}{3}, 1\right)$,

(e) $\left(w_{1}, w_{2}, w_{3}\right)=( \pm i,-1, \mp i),(-1, \pm i, \mp i)$.

Remark 1. Jørgensen [11, Theorem 8] characterized association schemes in Theorem1(i) in terms of a Bush-type Hadamard matrix of order $4 a^{2}$ whose off-diagonal blocks are skew-symmetric. Such a Bush-type Hadamard matrix can be constructed if there is a Hadamard matrix of order $2 a$ (see [12, Corollary 5 (ii)]). Note that, if $a$ is a power of 2, then such an association scheme can also be constructed from a Galois ring of characteristic 4 in [9, Theorem 9]. An association scheme with the first eigenmatrix in Theorem 1 (ii) is given by as08[6] in [5].

The organization of the paper is as follows. In Section 2, we consider matrices which belong to the Bose-Mesner algebra of a commutative association scheme, and we give a necessary and sufficient condition that such a matrix is a complex Hadamard matrix. We also introduce a result of S. Y. Song (14) which describes the eigenmatrices of nonsymmetric 3-class association schemes. In Section 3, we classify complex Hadamard matrices attached to self-dual fissions of a complete multipartite graph. In Section 4, we show that the Bose-Mesner algebra of an association scheme in the remaining cases of Song's description does not contain a complex Hadamard matrices.

All the computer calculations in this paper were performed with the help of Magma [2]. 


\section{Association SCHEMES AND COMPlEX HADAMARD MATRICES}

In this section, we consider matrices belonging to the Bose-Mesner algebra of a commutative association scheme. Assuming that all entries are complex numbers of absolute value 1, we find conditions under which such a matrix is a complex Hadamard matrix. We refer the reader to [1, 3] for undefined terminology in the theory of association schemes.

Let $X$ be a finite set with $n$ elements, and let $\left(X,\left\{R_{i}\right\}_{i=0}^{d}\right)$ be a commutative association scheme with the first eigenmatrix $P=\left(P_{i, j}\right)_{\substack{0 \leq i \leq d \\ 0 \leq j \leq d}}$. We let $\mathfrak{A}$ denote the Bose-Mesner algebra spanned by the adjacency matrices $A_{0}, A_{1}, \ldots, A_{d}$ of $\left(X,\left\{R_{i}\right\}_{i=0}^{d}\right)$. Then the adjacency matrices are expressed as

$$
A_{j}=\sum_{k=0}^{d} P_{k, j} E_{k} \quad(j=0,1, \ldots, d),
$$

where $E_{0}=\frac{1}{n} J, E_{1}, \ldots, E_{d}$ are the primitive idempotents of $\mathfrak{A}$. Set $m_{k}=\operatorname{rank} E_{k}$. Let $w_{0}=1$ and $w_{1}, \ldots, w_{d}$ be complex numbers of absolute value 1 . For each $j \in\{0,1, \ldots, d\}$, define $j^{\prime}$ by $A_{j^{\prime}}=A_{j}^{\top}$.

Set

$$
W=\sum_{j=0}^{d} w_{j} A_{j} \in \mathfrak{A} .
$$

Let $X_{0}=1$ and let $X_{j}(1 \leq j \leq d)$ be indeterminates. For $k=0,1, \ldots, d$, let $e_{k}$ be the polynomial in $X_{1}, \ldots, X_{d}$ defined by

$$
e_{k}=X_{1} \cdots X_{d}\left(\left(\sum_{j=0}^{d} P_{k, j} X_{j}\right)\left(\sum_{j=0}^{d} \frac{P_{k, j^{\prime}}}{X_{j}}\right)-n\right) \text {. }
$$

Lemma 1. The following statements are equivalent.

(i) the matrix $W$ defined by (4) is a complex Hadamard matrix,

(ii) the sequence $\left(w_{j}\right)_{1 \leq j \leq d}$ is a common zero of $e_{k}(k=0,1, \ldots, d)$.

Proof. By (3), (4) we have

$$
\begin{aligned}
W & =\sum_{k=0}^{d}\left(\sum_{j=0}^{d} w_{j} P_{k, j}\right) E_{k}, \\
\bar{W}^{\top} & =\sum_{j=0}^{d} \frac{1}{w_{j}} A_{j^{\prime}} \quad\left(A_{j^{\prime}}=A_{j}^{\top}\right) \\
& =\sum_{k=0}^{d}\left(\sum_{j=0}^{d} \frac{1}{w_{j}} P_{k, j^{\prime}}\right) E_{k} .
\end{aligned}
$$

By (6), (7) we have

$$
W \bar{W}^{\top}=\sum_{k=0}^{d}\left(\left(\sum_{j=0}^{d} w_{j} P_{k, j}\right)\left(\sum_{j=0}^{d} \frac{1}{w_{j}} P_{k, j^{\prime}}\right)\right) E_{k} .
$$

Therefore, the equivalence of (i) and (ii) follows. 
Let $\mathfrak{X}=\left(X,\left\{R_{i}\right\}_{i=0}^{3}\right)$ be a nonsymmetric 3 -class association scheme with the first eigenmatrix (11). Consider the polynomial ring

$$
R=\mathbb{C}\left[X_{1}, X_{2}, X_{3}\right]
$$

Then by (5) we have the following.

$$
\begin{aligned}
e_{0}= & \left(1+\frac{k_{1}}{2}\left(X_{1}+X_{2}\right)+k_{2} X_{3}\right)\left(X_{1} X_{2} X_{3}+\frac{k_{1}}{2}\left(X_{1}+X_{2}\right) X_{3}+k_{2} X_{1} X_{2}\right) \\
& -n X_{1} X_{2} X_{3} \\
e_{1}= & \left(1+\frac{r+b i}{2} X_{1}+\frac{r-b i}{2} X_{2}-(r+1) X_{3}\right) \\
& \times\left(X_{1} X_{2} X_{3}+\left(\frac{r+b i}{2} X_{1}+\frac{r-b i}{2} X_{2}\right) X_{3}-(r+1) X_{1} X_{2}\right) \\
& -n X_{1} X_{2} X_{3} \\
e_{2}= & e_{1}, \\
e_{3}= & \left(1+\frac{s}{2}\left(X_{1}+X_{2}\right)-(s+1) X_{3}\right) \\
& \times\left(X_{1} X_{2} X_{3}+\frac{s}{2}\left(X_{1}+X_{2}\right) X_{3}-(s+1) X_{1} X_{2}\right)-n X_{1} X_{2} X_{3} .
\end{aligned}
$$

Lemma 2. We have the following.

$$
\begin{aligned}
4\left(e_{0}-e_{3}\right)= & \left(2\left(k_{1} k_{2}+s^{2}+s\right)\left(X_{1}+X_{2}\right)+4\left(k_{2}+s+1\right) X_{1} X_{2}\right) X_{3}^{2} \\
+ & \left(k_{1}^{2}\left(X_{1}+X_{2}\right)^{2}+2\left(k_{1}-s\right)\left(X_{1} X_{2}+1\right)\left(X_{1}+X_{2}\right)\right. \\
& \left.-s^{2}\left(X_{1}^{2}+X_{2}^{2}\right)+2\left(2 k_{2}^{2}-3 s^{2}-4 s-2\right) X_{1} X_{2}\right) X_{3} \\
+ & 2\left(\left(k_{1} k_{2}+s(s+1)\right)\left(X_{1}+X_{2}\right)+2\left(k_{2}+s+1\right)\right) X_{1} X_{2}, \\
e_{1}-e_{2}=- & b i\left(X_{1}-X_{2}\right) \\
& \times\left((r+1)\left(X_{3}^{2}+X_{1} X_{2}\right)-\left(X_{1} X_{2}+r\left(X_{1}+X_{2}\right)+1\right) X_{3}\right) .
\end{aligned}
$$

Proof. Straightforward.

Lemma 3. Let $\left(w_{1}, w_{2}, w_{3}\right)$ be a common zero of the polynomials $e_{k}(k=0,1,2,3)$. Then $\left(w_{2}, w_{1}, w_{3}\right)$ is also a common zero of the polynomials $e_{k}$.

Proof. We have $e_{j}\left(X_{2}, X_{1}, X_{3}\right)=e_{j}\left(X_{1}, X_{2}, X_{3}\right)$ for $j=0,3$, and $e_{2}\left(X_{2}, X_{1}, X_{3}\right)=$ $e_{1}\left(X_{1}, X_{2}, X_{3}\right)$.

S. Y. Song [14, (5.3) Lemma] showed the following.

Lemma 4. Let $\left(1, m_{1}, m_{1}, m_{2}\right)$ denote the top row of the matrix $\left(1+k_{1}+k_{2}\right) P^{-1}$, where $P$ is given by (11). Then one of the following holds.

(i) $\left(r, s, b^{2}\right)=\left(0,-\left(k_{2}+1\right), \frac{k_{1}\left(k_{2}+1\right)}{k_{2}}\right), m_{1}=\frac{\left(k_{1}+k_{2}+1\right) k_{2}}{2\left(k_{2}+1\right)}$,

(ii) $\left(r, s, b^{2}\right)=\left(-\left(k_{2}+1\right), 0,\left(k_{2}+1\right)\left(k_{1}+k_{2}+1\right)\right), m_{1}=\frac{k_{1}}{2\left(k_{2}+1\right)}$,

(iii) $\left(r, s, b^{2}\right)=\left(-1, k_{1}, k_{1}+1\right), m_{1}=\frac{\left(k_{1}+k_{2}+1\right) k_{1}}{k_{1}+1}$.

In Lemma 4, (i) and (ii) are nonsymmetric fissions of a complete multipartite graph, (i) is self-dual, and (ii) is non self-dual. Also, (iii) is a nonsymmetric fission of a disjoint union of complete graphs. 
Lemma 5. Suppose that (i) in Lemma 4 holds. Define $a=-s / 2$. Then

$$
k_{2}=2 a-1,
$$

and $a$ is a positive integer. Moreover, there exists a positive integer $c$ such that

$$
\begin{aligned}
k_{1} & =2 a(2 a-1) c, \\
b & =2 a \sqrt{c} .
\end{aligned}
$$

Set

$$
L=(2 a-1) c^{2}-2(a+1) c-1 .
$$

Then we have the following.

(i) $L \neq 0$,

(ii) Suppose that $L<0$. Then one of the following holds.

(a) $c=1$,

(b) $(a, c)=(1,3)$, that is, $k_{1}=6, k_{2}=1, b=2 \sqrt{3}$.

Proof. Since $-a$ is an eigenvalue of the adjacency matrix $A_{1}, a$ is an integer. Since $s=-\left(k_{2}+1\right)$, we have (15). The symmetrization is the complete multipartite graph of the part size $k_{2}+1$. Thus, $k_{2}+1$ is a divisor of $n=1+k_{1}+k_{2}$, and hence $k_{2}+1$ is a divisor of $k_{1}$. Since $b^{2}=k_{1}\left(k_{2}+1\right) / k_{2}$ is an integer, $k_{2}$ is a divisor of $k_{1}$. Hence $k_{2}\left(k_{2}+1\right)$ is a divisor of $k_{1}$. Thus, there exists a positive integer $c$ such that (16) holds. Since $b$ is a positive real number, we have (17).

(i) Suppose that $L=0$. We regard (18) as a quadratic of $c$. Since the discriminant is $a(a+4)$, there exists a positive integer $m$ such that $a(a+4)=m^{2}$. Then $(a+2+m)(a+2-m)=4$. This contradicts a positive integer $a$.

(ii) Since $L<0$, we have

$$
1 \leq c \leq \frac{a+1+\sqrt{a(a+4)}}{2 a-1} .
$$

Then we have the following: If $a=1$ then $c \in\{1,2,3,4\}$. If $a=2$ then $c \in\{1,2\}$. If $a \geq 3$ then $c=1$. For $(a, c)=(1,2),(1,4),(2,2)$ we have $m_{1}=\frac{3}{2}, \frac{5}{2}, \frac{21}{2}$ by (i) in Lemma 4 respectively. Hence we have the assertions (a) or (b).

To conclude this section, we note that the matrices described in Theorem 1 are indeed complex Hadamard matrices. By Lemma 1, it suffices to show that $\left(w_{1}, w_{2}, w_{3}\right)$ is common zero of the polynomials (9)-(12), and it is easy to do this.

\section{Self-dual Fissions of a COMPlete multipartite GRAPH}

Throughout this section, we suppose that case (i) in Lemma 4 holds, and that the matrix (2) is a complex Hadamard matrix with $w_{1} \neq w_{2}$. By Lemma11 $\left(w_{1}, w_{2}, w_{3}\right)$ is a common zero of the polynomials $e_{k}(k=0,1,2,3)$.

Lemma 6. We have the following.

(i) $w_{3}=1$ or $w_{3}=w_{1} w_{2}$.

(ii) $w_{2}=-w_{1}$ or $g\left(w_{1}, w_{2}\right)=0$, where

$$
g\left(X_{1}, X_{2}\right)=2\left(X_{1} X_{2}+1\right)+((2 a-1) c-1)\left(X_{1}+X_{2}\right) .
$$

(iii) If $w_{1} w_{2}=1$, then $w_{2}=-w_{1}= \pm i$. 
Proof. After specializing $r=0, s=-2 a, k_{1}=2 a(2 a-1) c$, and $k_{2}=2 a-1$ in (13) and (14), we have

$$
\begin{aligned}
& e_{1}-e_{2}=b i\left(X_{1}-X_{2}\right)\left(X_{3}-1\right)\left(X_{1} X_{2}-X_{3}\right), \\
& e_{0}-e_{3}=a((2 a-1) c+1)\left(X_{1}+X_{2}\right) g_{1}\left(X_{1}, X_{2}, X_{3}\right),
\end{aligned}
$$

where

$$
\begin{aligned}
g_{1}\left(X_{1}, X_{2}, X_{3}\right)= & (2 a-1)\left(X_{1} X_{2}+X_{3}^{2}\right) \\
& +\left(X_{1} X_{2}+1+a((2 a-1) c+1)\left(X_{1}+X_{2}\right)\right) X_{3} .
\end{aligned}
$$

From (20) we have (i).

Observe, by (19) and (22),

$$
X_{1} X_{2} g_{1}\left(X_{1}, X_{2}, 1\right)=g_{1}\left(X_{1}, X_{2}, X_{1} X_{2}\right)=a X_{1} X_{2} g\left(X_{1}, X_{2}\right) .
$$

From (21) we have $w_{2}=-w_{1}$ or $g_{1}\left(w_{1}, w_{2}, w_{3}\right)=0$. Suppose that $g_{1}\left(w_{1}, w_{2}, w_{3}\right)=$ 0 . Since $w_{3}=1$ or $w_{3}=w_{1} w_{2}$ by (i), we have $g\left(w_{1}, w_{2}\right)=0$ by (23).

Finally, we suppose that $w_{1} w_{2}=1$. Then $w_{3}=1$ by (i). Suppose that $g\left(w_{1}, \frac{1}{w_{1}}\right)=0$. Then by (19) we have

$$
((2 a-1) c-1) w_{1}^{2}+4 w_{1}+(2 a-1) c-1=0 .
$$

Since $e_{1}\left(w_{1}, \frac{1}{w_{1}}, 1\right)=0$, we have

$$
a c w_{1}^{4}+2 a((a-1) c+1) w_{1}^{2}+a c=0 .
$$

From (24) and (25), by using the notation of (18), we have

$$
((2 a-1) c+1) L=0 .
$$

Since $a$ and $c$ are positive integers, this contradicts Lemma 5 (i). By (ii) we have $w_{2}=-w_{1}$, that is, $w_{1}= \pm i$. Therefore we have (iii).

Lemma 7. Suppose that $w_{2}=-w_{1}$. Then we have (a) in Theorem 1(i).

Proof. By (i) in Lemma 6 we have $w_{3}=1$ or $w_{3}=w_{1} w_{2}$.

First suppose that $w_{3}=1$. After specializing $X_{2}=-X_{1}$ and $X_{3}=1$, we have

$$
e_{1}=-2 a(c-1) X_{1}^{2} \text {. }
$$

Hence $c=1$. Therefore we have (a) in Theorem 1 (i).

Secondly suppose that $w_{3} \neq 1$. Then $w_{3}=w_{1} w_{2}$, that is, $w_{3}=-w_{1}^{2}$. After specializing $X_{2}=-X_{1}$ and $X_{3}=-X_{1}^{2}$, we have

$$
e_{1}=X_{1}^{2}\left(X_{1}^{4}+2((c-1) a+1) X_{1}^{2}+1\right) .
$$

Hence $w_{3}^{2}-2((c-1) a+1) w_{3}+1=0$. Since $a$ and $c$ are positive integers, we have $w_{3}=1$. This contradicts $w_{3} \neq 1$.

Lemma 8. Suppose that $w_{2} \neq-w_{1}$ and either $w_{1}$ or $w_{2}$ is -1 . Then $(a, c)=(2,1)$ or $(a, c)=(1,3)$.

Proof. We may set $w_{2}=-1$ without loss of generality. Since $w_{2} \neq-w_{1}$, by (ii) in Lemma 6 we have $g\left(w_{1}, w_{2}\right)=0$. Then by $w_{2}=-1$ we have $((2 a-1) c-3)\left(w_{1}-1\right)=$ 0 . By $w_{1} \neq 1$ we have $(2 a-1) c=3$. Since $a$ and $c$ are positive integers, we have the assertion.

Lemma 9. With the assumption in Lemma 8, suppose that $(a, c)=(2,1)$. Then we have (c) in Theorem 1 (i). 
Proof. We may set $w_{2}=-1$ without of loss of generality. By (15)-(17) we have $k_{1}=12, k_{2}=3$, and $b=4$. By (i) in Lemma 6 we have $w_{3}=1$ or $w_{3}=w_{1} w_{2}$.

First suppose that $w_{3}=1$. After specializing $X_{2}=-1, X_{3}=1, k_{1}=12$, and $k_{2}=3$, we have

$$
e_{1}=-4\left(X_{1}-1\right)^{2}
$$

Hence $w_{1}=1$. This contradicts $w_{1} \neq 1$.

Secondly suppose that $w_{3} \neq 1$. By (i) in Lemma 6 we have $w_{3}=w_{1} w_{2}$, that is, $w_{3}=-w_{1}$. After specializing $X_{2}=-1, X_{3}=-X_{1}, k_{1}=12$, and $k_{2}=3$, we have

$$
e_{1}=X_{1}\left(5 X_{1}^{2}-6 X_{1}+5\right) \text {. }
$$

Hence $5 w_{1}^{2}-6 w_{1}+5=0$, that is, $w_{1}=(3 \pm 4 i) / 5$. Together with the use of Lemma 31, we have (c) in Theorem 1 (i).

Lemma 10. With the assumption in Lemma 8 , suppose that $(a, c)=(1,3)$. Then we have (d) or (e) in Theorem 1 (ii).

Proof. We may set $w_{2}=-1$ without of loss of generality. By (15)-(17) we have $k_{1}=6, k_{2}=1$, and $b=2 \sqrt{3}$. By (i) in Lemma 6 we have $w_{3}=1$ or $w_{3}=w_{1} w_{2}$.

First suppose that $w_{3}=1$. After specializing $X_{2}=-1, X_{3}=1, k_{1}=6$, and $k_{2}=1$, we have

$$
e_{1}=-\left(3 X_{1}^{2}-2 X_{1}+3\right)
$$

Hence $3 w_{1}^{2}-2 w_{1}+3=0$, that is, $w_{1}=(1 \pm 2 \sqrt{2} i) / 3$. Together with the use of Lemma 3, we have (d) in Theorem 1 (ii).

Secondly suppose that $w_{3}=w_{1} w_{2}$, that is, $w_{3}=-w_{1}$. After specializing $X_{2}=$ $-1, X_{3}=-X_{1}, k_{1}=6$, and $k_{2}=1$, we have

$$
e_{1}=4 X_{1}\left(X_{1}^{2}+1\right) \text {. }
$$

Hence $w_{1}= \pm i$. Together with the use of Lemma 3, we have (e) in Theorem 1 (ii).

Lemma 11. Suppose that $w_{2} \neq-w_{1}$ and $w_{1}, w_{2} \neq \pm 1$. Then we have (b) in Theorem 1(i).

Proof. By (iii) in Lemma 6 we have $w_{1} w_{2} \neq 1$, while $w_{1} \neq w_{2}$ by our assumption. This means that $w_{1}, \overline{w_{1}}, w_{2}, \overline{w_{2}}$ are mutually distinct. Let $x_{j}=w_{j}+1 / w_{j}$ for $j=1,2$. Then the polynomial having $w_{1}, \overline{w_{1}}, w_{2}, \overline{w_{2}}$ as roots is given by

$$
f(X)=\left(X^{2}-x_{1} X+1\right)\left(X^{2}-x_{2} X+1\right) .
$$

By the assumption $w_{2} \neq-w_{1}$ and (ii) of Lemma 6 we have $g\left(w_{1}, w_{2}\right)=0$. Let $\mathcal{J}$ denote the ideal of (8) generated by the polynomials $e_{k}(k=0,1,2,3)$ and (19). By (i) in Lemma 6 we have $w_{3}=1$ or $w_{3}=w_{1} w_{2}$.

First suppose that $w_{3}=1$. Let $\mathcal{J}_{1}$ denote the ideal of (8) generated by $\mathcal{J}$ and $X_{3}-1$. Then $\left(w_{1}, w_{2}, 1\right)$ is a common zero of polynomials in $\mathcal{J}_{1}$. We can verify that $\mathcal{J}_{1}$ contains $f_{1}\left(X_{1}\right), f_{1}\left(X_{2}\right)$, where

$$
\begin{aligned}
f_{1}(X) & =a_{0} X^{4}+a_{1} X^{3}+a_{2} X^{2}+a_{1} X+a_{0}, \\
a_{0} & =2 a c, \\
a_{1} & =2(c-1)((2 a-1) c-1), \\
a_{2} & =4 a c^{2}((c-1) a-c)+(c-1)\left(c^{2}+2 c+5\right) .
\end{aligned}
$$


Then $f_{1}\left(w_{1}\right)=f_{1}\left(w_{2}\right)=0$. Since $f_{1}(X)$ has real coefficients, we have $f_{1}(X)=$ $a_{0} f(X)$. Comparing the coefficients of (26) and (27), we have

$$
\begin{aligned}
a_{0}\left(x_{1}+x_{2}\right)+a_{1} & =0, \\
a_{0} x_{1} x_{2}+2 a_{0}-a_{2} & =0 .
\end{aligned}
$$

By (28) and (29), $x_{1}$ and $x_{2}$ are the roots of

$$
a_{0} X^{2}+a_{1} X-2 a_{0}+a_{2}=0 .
$$

Then by using the notation of (18), since the discriminant of (30) is

$$
-((2 a-1) c+1)^{2} L
$$

and $x_{1} \neq x_{2}$, we have $L<0$. First we consider (a) of Lemma 5 (ii). Then by (30) we have $X^{2}-4=0$, that is, $w_{1}, w_{2} \in\{ \pm 1\}$. This contradicts our assumption. Secondly we consider (b) in Lemma 5 (ii). Then, (28) and (29) are $3\left(x_{1}+x_{2}\right)+4=0$ and $3 x_{1} x_{2}+4=0$, respectively. Then $\left\{x_{1}, x_{2}\right\}=\left\{-2, \frac{2}{3}\right\}$, that is, either $w_{1}$ or $w_{2}$ is -1 . This contradicts our assumption.

Secondly suppose that $w_{3}=w_{1} w_{2} \neq 1$. Let $\mathcal{J}_{2}$ denote the ideal of (8) generated by $\mathcal{J}$ and $X_{3}-X_{1} X_{2}$. Then $\left(w_{1}, w_{2}, w_{1} w_{2}\right)$ is a common zero of the polynomials in $\mathcal{J}_{2}$. We can verify that $\mathcal{J}_{2}$ contains $X_{i}\left(((2 a-1) c-1) X_{i}+2\right) f_{2}\left(X_{i}\right), i=1,2$, where

$$
\begin{aligned}
f_{2}(X) & =b_{0} X^{4}+b_{1} X^{3}+b_{2} X^{2}+b_{1} X+b_{0}, \\
b_{0} & =(c+1)(4 a c(a-1)+c+1), \\
b_{1} & =4(a(c-1)+2)((2 a-1) c-1), \\
b_{2} & =2 a(2 a-1)^{2} c^{3}-2(2 a-1)\left(2 a^{2}-a+1\right) c^{2}-2(a-2) c-2(5 a-9) .
\end{aligned}
$$

Then $f_{2}\left(w_{1}\right)=f_{2}\left(w_{2}\right)=0$. Since $f_{2}(X)$ has real coefficients, we have $f_{2}(X)=$ $b_{0} f(X)$. Comparing coefficients of (26) and (31), we have

$$
\begin{aligned}
& b_{0}\left(x_{1}+x_{2}\right)+b_{1}=0, \\
& b_{0} x_{1} x_{2}+2 b_{0}-b_{2}=0 .
\end{aligned}
$$

By (32) and (33) $x_{1}$ and $x_{2}$ are the roots of

$$
b_{0} X^{2}+b_{1} X-2 b_{0}+b_{2}=0 .
$$

Then by using the notation of (18), the discriminant of (34) is

$$
-2 a((2 a-1) c+1)^{2}((2 a-1) c+2 a-3) L .
$$

Since $x_{1} \neq x_{2}$, we have $(2 a-1) c+2 a-3 \neq 0$ and $L<0$.

First we consider (a) in Lemma 5 (ii). Then by (34) the real parts of $w_{1}$ and $w_{2}$ are given by

$$
\frac{-a+1 \pm a \sqrt{2 a(a-1)}}{2 a^{2}-2 a+1} .
$$

Since $\left|w_{1}\right|=\left|w_{2}\right|=1$, we have (b) in Theorem 1 (i).

Secondly we consider (b) in Lemma 5 (ii). Then by $a=1$ and $c=3$, (34) is $X(X+2)=0$, that is, either $w_{1}$ or $w_{2}$ is -1 . This contradicts our assumption.

Therefore, we have shown that, in case (i) in Lemma 4, complex Hadamard matrices must arise in one of the ways described in Theorem 1. In the next section, we will show the nonexistence of a complex Hadamard matrices for cases (ii) and (iii) in Lemma 4 


\section{OTHER CASES}

In this section we consider cases (ii) and (iii) in Lemma 4

Lemma 12. Suppose that the matrix (1) satisfies case (ii) in Lemma 4, Then there does not exist complex Hadamard matrices of the form (2).

Proof. After specializing $r=-\left(k_{2}+1\right)$ and $s=0$, we have

$$
-e_{3}=X_{1} X_{2}\left(X_{3}^{2}+\left(k_{1}+k_{2}-1\right) X_{3}+1\right) \text {. }
$$

Hence

$$
w_{3}^{2}+\left(k_{1}+k_{2}-1\right) w_{3}+1=0
$$

Since $k_{1}+k_{2}-1 \geq 2$, we have $w_{3}=-1$ and $k_{1}+k_{2}=3$. Since $k_{1}$ is even, we have $k_{1}=2$ and $k_{2}=1$. Then by (ii) in Lemma 4 we have $m_{1}=\frac{1}{2} \notin \mathbb{Z}$. This is a contradiction.

Lemma 13. Assume that the matrix (1) satisfies case (iii) in Lemma 4. Then there does not exist complex Hadamard matrices of the form (2).

Proof. After specializing $r=-1$ and $s=k_{1}$ in (13) and (14), we have

$$
\begin{aligned}
& e_{0}-e_{3}=\frac{1}{2}\left(k_{1}+k_{2}+1\right) h_{1}\left(X_{1}, X_{2}, X_{3}\right), \\
& e_{1}-e_{2}=b i X_{3}\left(X_{1}-X_{2}\right)\left(X_{1}-1\right)\left(X_{2}-1\right),
\end{aligned}
$$

where

$$
\begin{aligned}
h_{1}\left(X_{1}, X_{2}, X_{3}\right)= & k_{1}\left(X_{1}+X_{2}\right)\left(X_{1} X_{2}+X_{3}^{2}\right)-2\left(k_{1}-k_{2}+1\right) X_{1} X_{2} X_{3} \\
& +2 X_{1} X_{2}\left(X_{3}^{2}+1\right) .
\end{aligned}
$$

Then by (36) we have

$$
h_{1}\left(w_{1}, w_{2}, w_{3}\right)=0 .
$$

We may set $w_{1}=1$ without of loss of generality by (37). After specializing $X_{1}=1$, $r=-1$, and $s=k_{1}$, we have

$$
-4 e_{1}=X_{3}\left(\left(k_{1}+2\right) X_{2}^{2}+2\left(k_{1}+2 k_{2}\right) X_{2}+k_{1}+2\right) .
$$

Hence

$$
w_{2}^{2}+\frac{2\left(k_{1}+2 k_{2}\right)}{k_{1}+2} w_{2}+1=0 .
$$

Since $\frac{2\left(k_{1}+2 k_{2}\right)}{k_{1}+2} \geq 2$, we have $w_{2}=-1$. Then by (39) we have $k_{2}=1$. Then by (iii) in Lemma 4 we have $m_{1}=\frac{\left(k_{1}+2\right) k_{1}}{k_{1}+1} \notin \mathbb{Z}$. This is a contradiction.

\section{REFERENCES}

[1] E. Bannai and T. Ito, Algebraic Combinatorics I: Association Schemes, Benjamin/Cummings, Menlo Park, 1984.

[2] W. Bosma, J. Cannon, and C. Playoust, The Magma algebra system. I. The user language, J. Symbolic Comput., 24 (1997), 235-265.

[3] A. E. Brouwer, A. M. Cohen, and A. Neumaier, Distance-Regular Graphs, Springer-Verlag, Berlin, Heidelberg, 1989.

[4] A. Chan and C. Godsil, Type-II matrices and combinatorial structures, Combinatorica, 30 (2010), 1-24.

[5] A. Hanaki, Classification of association schemes with small vertices, http://math.shinshu-u.ac.jp/ hanaki/as/

[6] A. Hanaki and I. Miyamoto, Classification of association schemes with 16 and 17 vertices, Kyushu J. Math. 52 (2), (1998) 383-395. 
[7] A. Hanaki and I. Miyamoto, Classification of association schemes with 18 and 19 vertices, Korean J. Comput. Appl. Math. 5 (3), (1998) 543-551.

[8] T. Ikuta and A. Munemasa, Butson-type complex Hadamard matrices and association schemes on Galois rings of characteristic 4, Spec. Matrices, 6 (1), (2018) 1-10.

[9] T. Ito, A. Munemasa, and M. Yamada, Amorphous association schemes over the Galois rings of characteristic 4, European J. Combin., 12 (1991), 513-526.

[10] L. K. Jørgensen, G. A. Jones, M. H. Klin, and S. Y. Song, Normally regular digraphs, association schemes and related combinatorial structures, Sém. Lothar. Combin. 71 (2014), Article B71c, 1-39.

[11] L. K. Jørgensen, Algorithmic approach to non-symmetric 3-class association schemes, Algorithmic Algebraic Combinatorics and Gröbner Bases, 251-268, Springer, Berlin, 2009.

[12] H. Kharaghani, New class of weighing matrices, Ars Combin. 19 (1985), 69-72.

[13] A. Munemasa and Y. Watatani, Paires orthogonales de sous-algèbres involutives, C. R. Acad. Sci. Paris, Sér. I, 314 (1992), 329-331.

[14] S. Y. Song, Class 3 association schemes whose symmetrizations have two classes, J. Combin. Theory, Ser. A, 70 (1995) 1-29.

[15] F. Szöllősi, Construction, classification and parametrization of complex Hadamard matrices, Ph.D Thesis, Central European University, Budapest (2012).

[16] Wallis, W. D. On a problem of K. A. Bush concerning Hadamard matrices, Bull. Austral. Math. Soc. 6 (1972), 321-326.

\section{Appendix A. Verification by Magma}

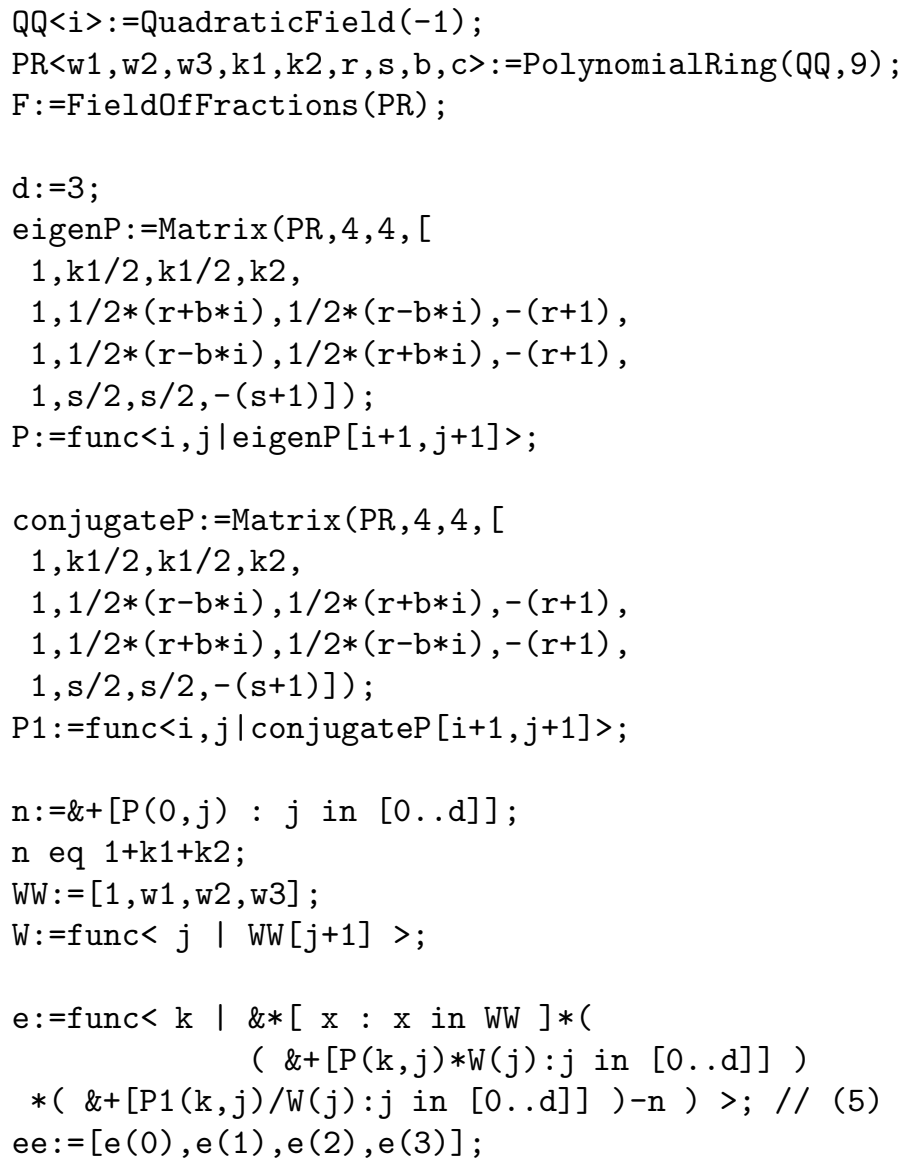




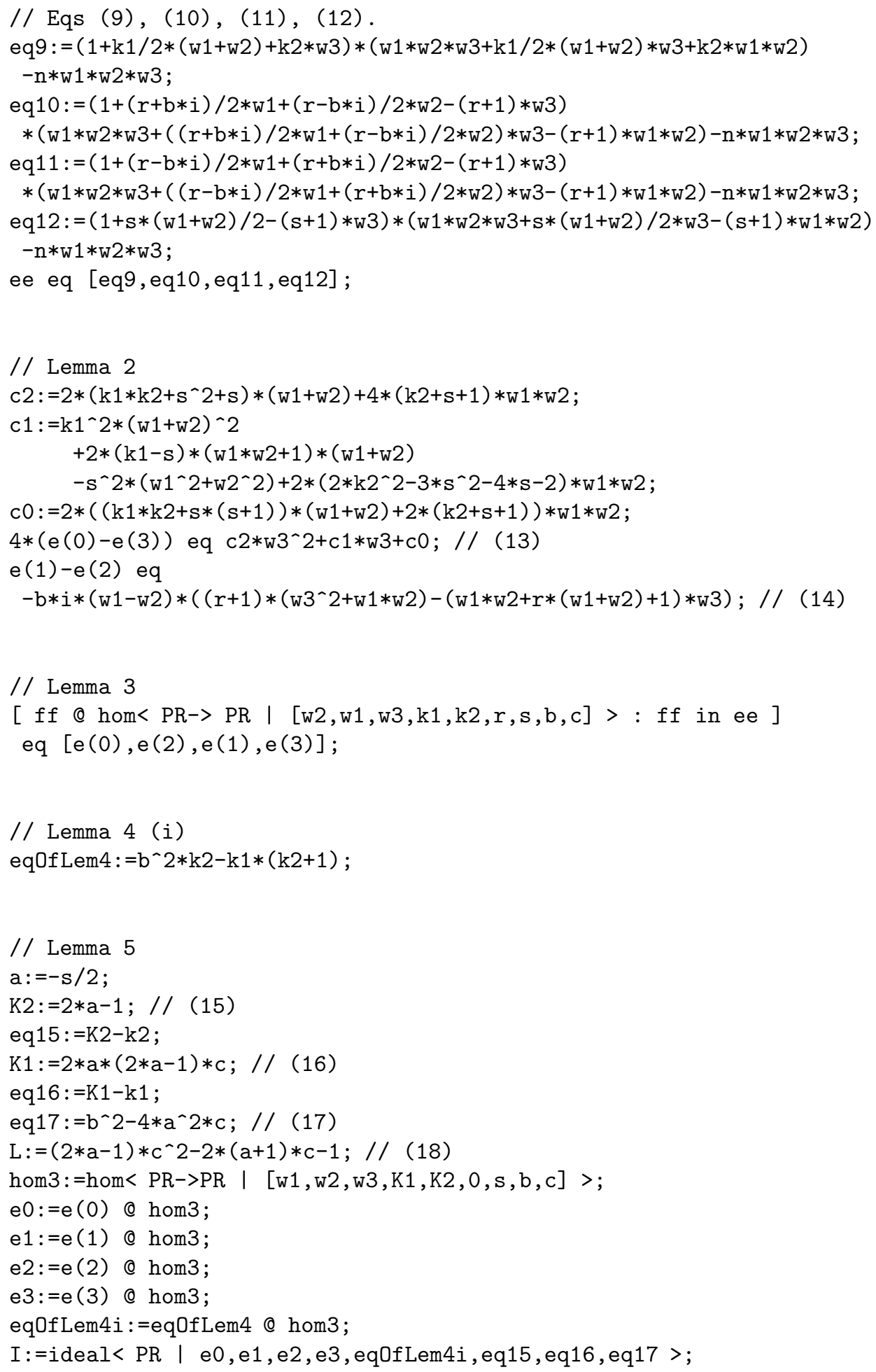




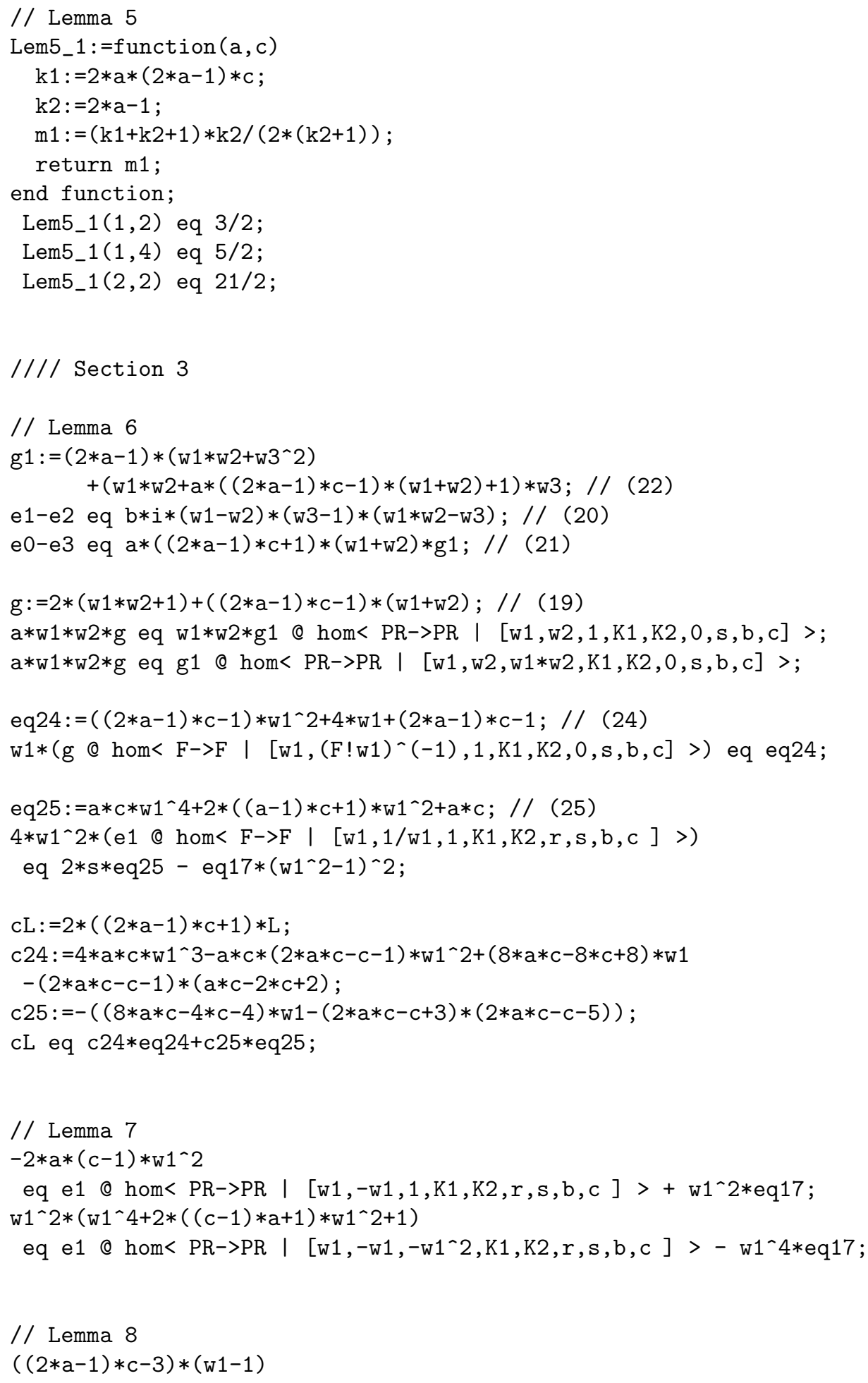


eq $g @$ hom< PR->PR | $[\mathrm{w} 1,-1, \mathrm{w} 3, \mathrm{~K} 1, \mathrm{~K} 2, \mathrm{r}, \mathrm{s}, \mathrm{b}, \mathrm{c}]>$;

// Lemma 9, $(\mathrm{a}, \mathrm{c})=(2,1)$, that is, $(\mathrm{s}, \mathrm{c})=(-4,1)$

12 eq K1 @ hom $<\mathrm{PR}->\mathrm{PR} \mid[\mathrm{w} 1,-1, \mathrm{w} 3, \mathrm{~K} 1, \mathrm{~K} 2, \mathrm{r},-4,4,1]>$;

3 eq $\mathrm{K} 2 @ \mathrm{hom}<\mathrm{PR}->\mathrm{PR} \mid[\mathrm{w} 1,-1, \mathrm{w} 3, \mathrm{~K} 1, \mathrm{~K} 2, \mathrm{r},-4,4,1]>$;

$-4 *(\mathrm{w} 1-1)^{\wedge} 2$ eq e1 @ hom< PR->PR | [w1,-1,1,K1,K2,r, -4,4,1] >;

$\mathrm{w} 1 *(5 * \mathrm{w} 1 \sim 2-6 * \mathrm{w} 1+5)$ eq

e1@ hom< PR->PR | [w1,-1,-w1,K1,K2,r, -4,4,1] >;

// Lemma 10, $(\mathrm{a}, \mathrm{c})=(1,3)$, that is, $(\mathrm{s}, \mathrm{c})=(-2,3)$

6 eq $\mathrm{K} 1$ @ hom $<\mathrm{PR}->\mathrm{PR} \mid[\mathrm{w} 1,-1, \mathrm{w} 3, \mathrm{~K} 1, \mathrm{~K} 2, \mathrm{r},-2, \mathrm{~b}, 3]>$;

1 eq $\mathrm{K} 2$ @ hom< PR->PR | [w1,-1, w3, K1, K2, r, -2, b, 3 ] >;

eqOfLem101: =- $(3 * w 1-2-2 * w 1+3)$;

eq0fLem101-1/4*(b^2-12)*(w1+1)^2

eq e1@ hom< PR->PR | [w1,-1,1,K1,K2,r, -2, b, 3 ] >;

eqOfLem102: $=4 * w 1 *(w 1 ` 2+1)$;

eq0fLem102 $+1 / 4 *\left(b^{\wedge} 2-12\right) * w 1 *(w 1+1)^{\wedge} 2$

eq e1@ hom< PR->PR | [w1,-1,-w1,K1,K2,r, -2, b, 3 ] >;

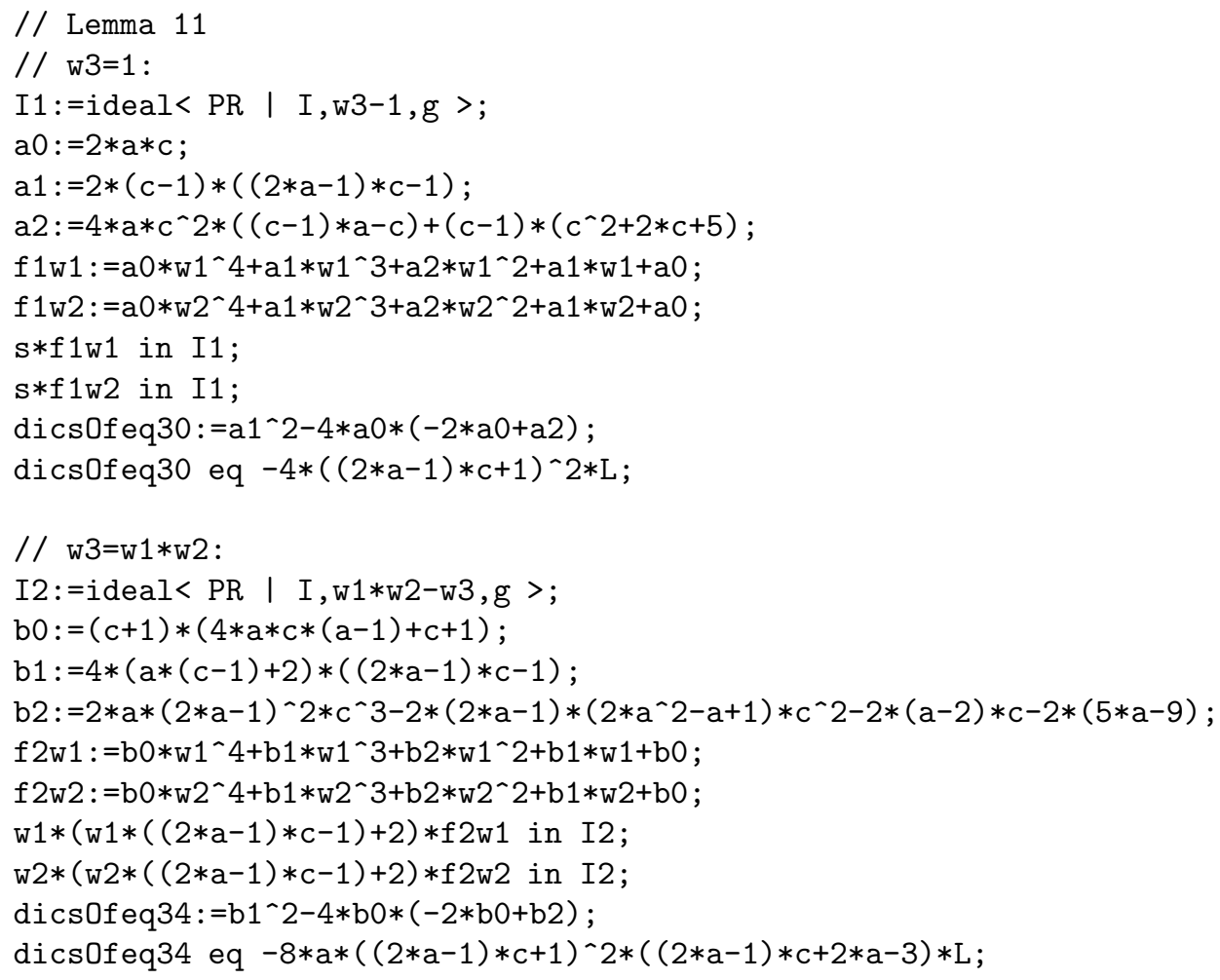




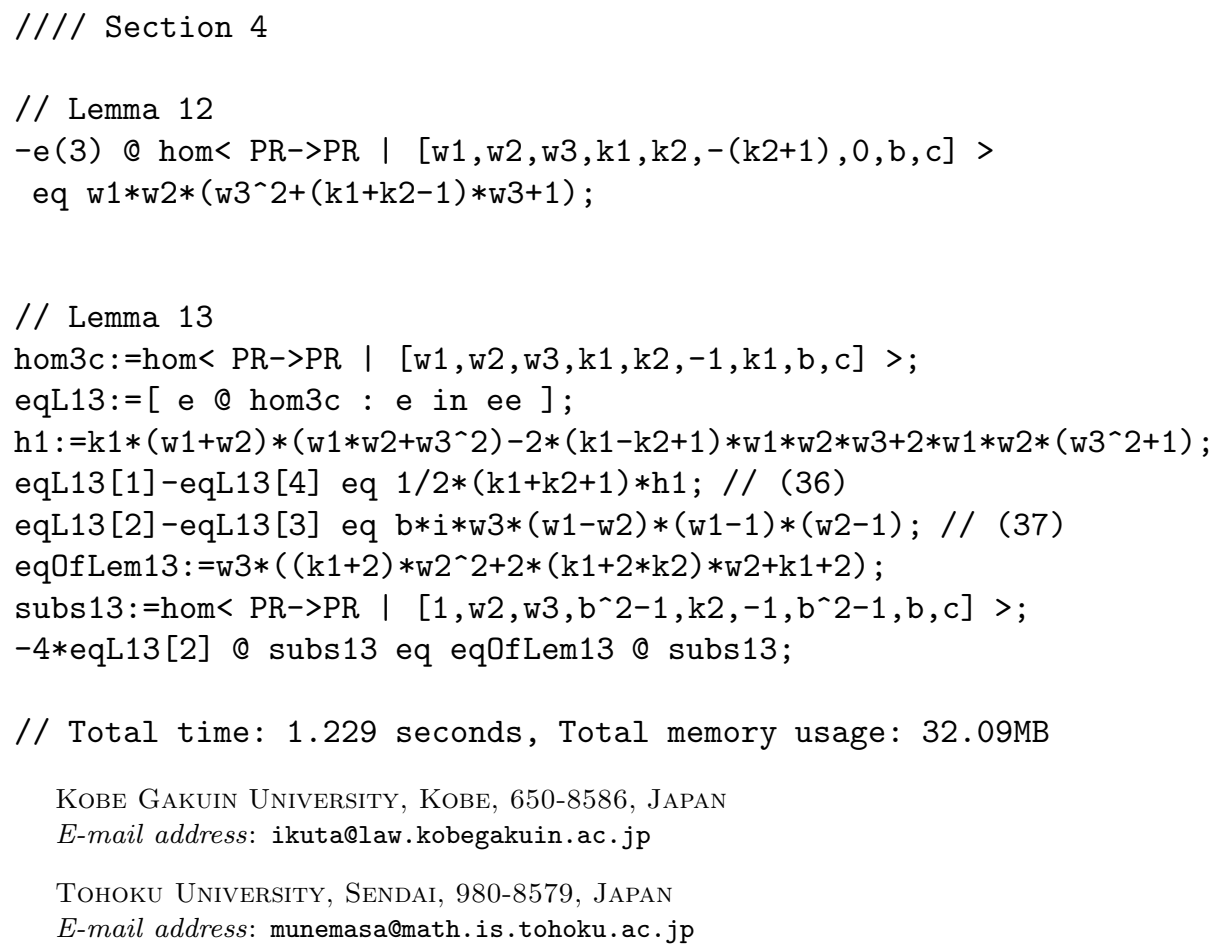

